\title{
Macroscopic Cross-Diffusion Models Derived From Spatially Discrete Continuous Time Microscopic Models*
}

\author{
Stephen Ostrander \\ McMaster University, Hamilton, ON, Canada, \\ email: ostransj@mcmaster.ca \\ Faculty mentor: \\ Hermann J. Eberl, University of Guelph, Guelph, ON, Canada \\ email: heberl@uoguelph.ca
}

May 1, 2011

\begin{abstract}
We formulate a continuous time, discrete in space model for two spatially interacting species. The spatial interaction is described in terms of a measure for the desire or ability of a population to move from one location into a neighboring site. This can depend on local densities of both populations in the current and the target site. Refining the spatial resolution and passing to a continuous in space model, one obtains a system of partial differential equations with cross diffusion terms. We show that certain cross-diffusion models that have been used in the literature to describe interacting species can be derived as special cases with our approach.
\end{abstract}

\section{Introduction}

In Mathematical Biology and in Theoretical Ecology, the spatial dispersal of populations is frequently described by dividing the space into discrete sites and modeling the movement of the population based on the probability that an individuum moves from the site it currently occupies into a neighboring site $[1,4,5,6,7,9,10]$. This is often referred to as a microscopic description because the pattern of movement of the population emerges from individuum level assumptions. For single species systems, macroscopic, population level,

${ }^{*}$ This research is the results of an undergraduate research assistantship in the Computational Biomathematics Laboratory at the University of Guelph during Summer 2010 
models in the form of partial differential equations are often derived from such microscopic models by refining the spatial discretisation more and more and by passing to the continuous limit.

In this paper, we will present a similar approach to derive macroscopic models for biological or ecological systems in which two interacting species occupy the same spatial environment. We start with micro-level assumptions about the probability of individuals to move from one discrete site into another one, depending on the current population densities of both species in both sites. Performing the same steps that are usually taken in modeling single species systems, we derive a general macroscopic model consisting of two partial differential equations with cross-diffusion.

While several of these PDE models have been used in the literature to describe interacting populations, they are to the best of our knowledge ad hoc and usually not derived from a description of microscopic behavior. Using our microscale description we will give such a derivation for two cross-diffusion models, namely the Shigesada-Kawasaki-Teramoto model [8] and the ChattopadhyayChatterjee model [2]. This is the primary goal of this study.

\section{A microscopic continuous time, discrete in space model for two interacting populations}

Our starting point is a microscale master equation model for the movement of two interacting populations. Models of this type have been studied extensively to model the dispersal of single populations $[1,4,5,6,7,9,10]$. We formulate this in a one-dimensional context but point out that the generalization to the twodimensional or three-dimensional setting is straightforward, but algebraically more involved.

Our spatial environment is represented by discrete non-overlapping sites, or locations, $x_{i}$ where $i \in \mathbb{Z}$. Since we are considering only finite environments, we use $i=1, \ldots ., n$. The densities of both populations in the $i$ th site are denoted by $u_{i}$ and $v_{i}$. They can vary in time. Therefore, $u_{i}(t)$ and $v_{i}(t)$ can be considered grid or lattices functions. We treat time $t$ as a continuous variable.

Rules governing the movements of the two species are then formulated, based on microscopic behavior on the level of the individuum. These rules take into account the population density of each species at both the site a population currently resides in (the departure site), and the site the population is moving into (the arrival or target site). The master equation describes the population change of a particular species in a particular site, by balancing the amount of the population which leaves the site to move into neighboring locations, and the amount which arrives from neighboring sites. The difference between these amounts is the population change of the species in the site.

The master equations for two interacting species read 


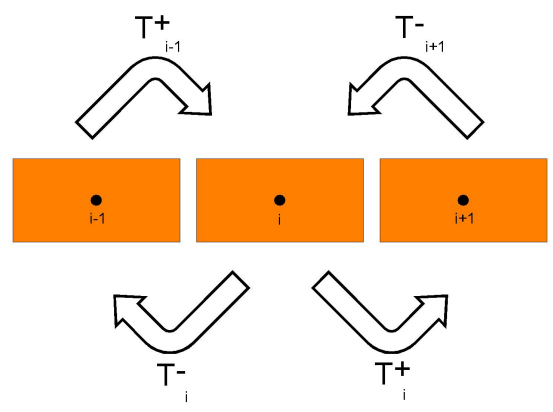

(a) species $u$

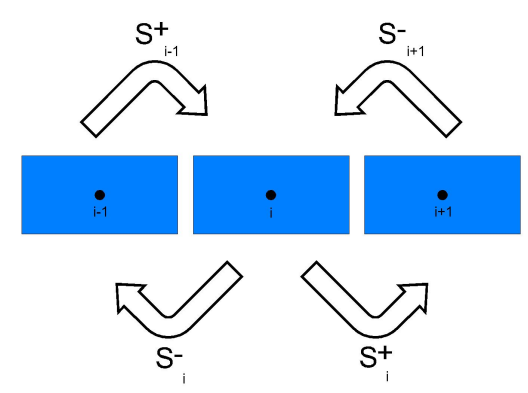

(b) species $\mathrm{v}$

Figure 1: Schematic of movements of populations between neighboring lattice cells. The flux of species $u$ that moves from cell $i$ to cell $i+1$ is denoted $\mathcal{T}_{i}^{+}$, etc.

$$
\left\{\begin{aligned}
\frac{d u_{i}}{d t} & =\mathcal{T}_{i-1}^{+} u_{i-1}+\mathcal{T}_{i+1}^{-} u_{i+1}-\left(\mathcal{T}_{i}^{+}+\mathcal{T}_{i}^{-}\right) u_{i}+\mathcal{K}_{i}^{u} \\
\frac{d v_{i}}{d t} & =\mathcal{S}_{i-1}^{+} v_{i-1}+\mathcal{S}_{i+1}^{-} v_{i+1}-\left(\mathcal{S}_{i}^{+}+\mathcal{S}_{i}^{-}\right) v_{i}+\mathcal{K}_{i}^{v}
\end{aligned}\right.
$$

where $\mathcal{T}_{i}^{ \pm}$and $\mathcal{S}_{i}^{ \pm}$are the rates at which species $u$ and $v$ move from the $i$ th site into the neighboring sites $i \pm 1$, cf also Figure 1 .

These dispersal rates in general can depend on the density of both populations in sites $i$ and $i \pm 1$. To account for this we make the general ansatz

$$
\begin{aligned}
& \mathcal{T}_{i}^{ \pm}=\alpha q\left(u_{i}, v_{i}\right) p\left(u_{i \pm 1}, v_{i \pm 1}\right) \\
& \mathcal{S}_{i}^{ \pm}=\beta r\left(u_{i}, v_{i}\right) m\left(u_{i \pm 1}, v_{i \pm 1}\right) .
\end{aligned}
$$

Here the non-negative functions $q, p, r, m$ are the "jump probabilities" which 
control the local movements of a species from one site on the lattice to a neighboring site and are taken to be continuous. The function $q\left(u_{i}, v_{i}\right)$ is a measure of the desire of species $u$ to leave the cell it is currently in $\left(x_{i}\right)$, while $p\left(u_{i}, v_{i}\right)$ represents the favorableness of the cell $x_{i}$ for the incoming individuals of species $u$. The functions $r\left(u_{i}, v_{i}\right)$ and $m\left(u_{i}, v_{i}\right)$ have identical meanings, but for species $v$.

These functions can be understood as probabilities for an individuum to move between sites $i$ and $i \pm 1$. The coefficient $\alpha$ is a scaling factor that depends on time-scale and length-scale, i.e. distance between two sites $h$. In dispersal lattice models of this kind, $\alpha$ scales with $h^{2}$, such that $\lim _{h \rightarrow 0} \alpha h^{2}=\alpha_{0}>0$. For a given grid $\alpha_{0}$ is a constant. The same applies for $\beta$.

The terms $\mathcal{K}_{i}^{u}$ and $\mathcal{K}_{i}^{v}$ in (1) are the net growth terms for species $u$ and $v$ respectively. These growth terms can be used to describe local interaction of both species, such as predator prey dynamics, competition for a substrate, and many others. The focus of our study is on the spatial dispersal. Therefore, we will always simplify and take $\mathcal{K}_{i}^{u}=\mathcal{K}_{i}^{v}=0$.

As a demonstration of the application of this model, several combinations of jump probabilities representing different dispersal strategies of the two species are considered and illustrated by computer simulations (Figures 2, 3). The simulations are run using Matlab where the differential equations are solved numerically by the Runge-Kutta 45 method.

All simulations are run on a finite lattice consisting of 100 cells. On the first and last cell, zero-flux boundary conditions are applied at $i=1$ and $i=$ 100 which compare to homogeneous Neumann conditions in partial differential equations. These boundary conditions are implemented by adjusting (1) in such a way that no net flow of populations takes place across the lattice boundaries. This is done by creating "ghost cells" at $i=0$ and $i=101$ such that $u_{0}=u_{1}$, $v_{0}=v_{1}, u_{100}=u_{101}$, and $v_{100}=v_{101}$ for all time. Also, since all cells on the lattice are considered to have volume equal to one and $\mathcal{K}_{i}^{u}=\mathcal{K}_{i}^{v}=0$, the total mass of a species $\sum_{i} u_{i}(t)$ is constant by these boundary conditions (likewise for $v$ ).

All the simulations given in this paper eventually lead to spatially and temporally homogeneous equilibriums, but with different transient behaviors. These transient behaviors are of the most interest in the context of this paper as they show how different dispersal strategies, expressed in terms of the jump probabilities, affect the movements of the populations differently.

Each simulation is also run with the same initial conditions. At time zero, species $u$ and $v$ are organized in one pocket each, separated around the middle of the lattice. Initially, each species is spread across 20 sites. The two populations are separated by 10 empty cells. The exact initial conditions are

$$
\left\{\begin{array}{l}
u_{i}(0)=0.5,26 \leq i \leq 45 \\
u_{i}(0)=0,0 \leq i \leq 25,46 \leq i \leq 100 \\
v_{i}(0)=0.5,56 \leq i \leq 75 \\
v_{i}(0)=0,0 \leq i \leq 55,76 \leq i \leq 100
\end{array}\right.
$$


In the first model, we mimic the dispersal strategies that one might encounter in predator-prey interaction, where species $u$ represents the prey population and $v$ the predator population. The jump probabilities used are

$$
\left\{\begin{array}{l}
p(u, v)=e^{-5 v} \\
q(u, v)=1-\frac{4}{5} e^{-5 v} \\
m(u, v)=1-\frac{4}{5} e^{-5 u} \\
r(u, v)=e^{-5 u}
\end{array}\right.
$$

These functions relate to the prey seeking locations with low predator density $\left(p_{v}<0, q_{v}>0\right)$ and diffusing randomly in the absence of predators $(p(u, 0)=1$, $q(u, 0)=0.2)$, and predators seeking locations with high prey density $\left(m_{u}>0\right.$, $\left.r_{u}<0\right)$ and randomly diffusing in the absence of prey $(m(0, v)=0.2, r(0, v)=$ 1). An alternative, qualitatively similar model, again with $u$ representing the prey species and $v$ the predator, but with linear jump probabilities is

$$
\left\{\begin{array}{l}
p(u, v)=1-v, \\
q(u, v)=\frac{1}{10}+\frac{9}{10} v \\
m(u, v)=\frac{1}{2}+\frac{1}{2} u \\
r(u, v)=1-u .
\end{array}\right.
$$

In the simulations (Figure 2) of these two models, we see similar results. The two populations spread randomly at a constant rate until they meet. This describes random searching for food. Once they encounter each other, the predator population's preference to reside in cells with the highest prey populations takes over. The predator population moves towards the prey, and the prey respond by moving to the left on the grid, away from the predators. This process is much more pronounced in the case of exponential jump probabilities as they change much faster at low concentrations of $u$ and $v$ than functions in the linear chase scenario. It is important to note that the location of the peak of the predator population does not change notably in either the linear or exponential chase scenarios. This is because the populations located in these cells have not been in contact with any prey population yet. The degree to which individuals change their behavior depends on how much interaction they have with the other species and a population of a species can only "see" a population of another species if they are located in the same cell or neighboring cell.

In the first model, with exponential jump probabilities, once the prey population has moved to the left of the grid (Figure 2(a)), there is nowhere left to "run" from the predators. The predator density then begins to increase in this location. This causes the prey population to diffuse out of the corner of the grid. This process eventually leads to the prey slightly outnumbering predators on the right hand side of the lattice and vice versa on the left hand side as shown in Figure 2(b). The populations then continue diffusing towards a spatially and temporally homogenous equilibrium (data not shown).

In the first two examples, the dispersal strategy of a species changed in response to the density of the other species but it was independent of its own 


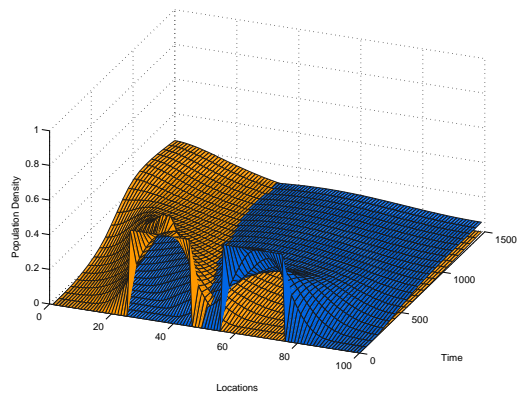

(a) Exponential Chase Scenario

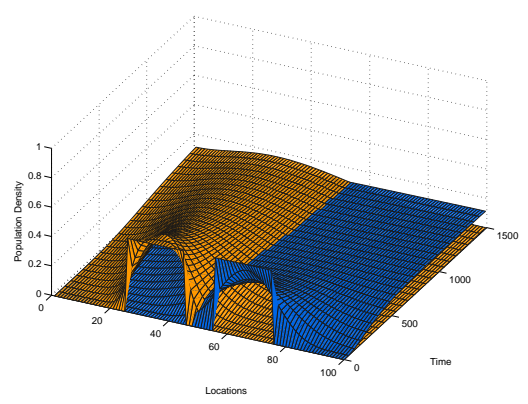

(c) Linear Chase

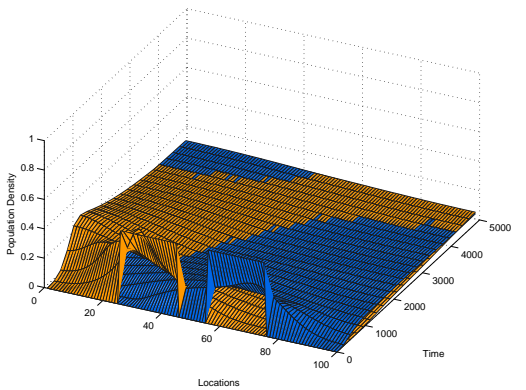

(b) Exponential Chase (extended)

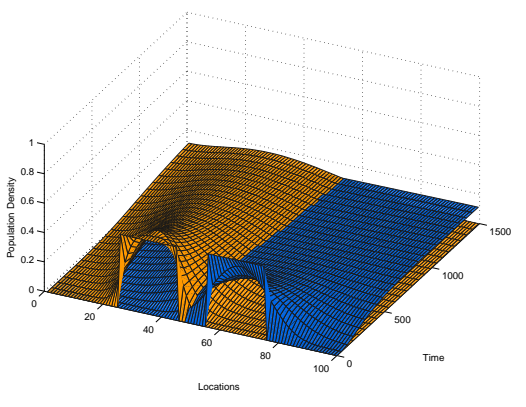

(d) Linear Chase (extended)

Figure 2: Simulations using jump probabilities from Section 2. Species $v$ is shaded blue and species $u$ is orange. Figures (a),(b): jump probabilities given by (3), 300 and 1500 time steps, respectively. Figures (c), (d): jump probabilites from (4), 300 and 1500 time steps, respectively.

density. The next model compares the diffusion pattern of two spatially noninteracting species, where the dispersal strategy of species $u$ only depends on the density of its own species in the arrival and target site, but not on the density of the other population. This is an example of a system where the cross diffusion effect is elminated as well as a demonstration of an aggregation strategy, with species $u$ preferentially seeking sites with higher concentrations of its own species (up to a density of 0.5 ) while also randomly diffusing at a slow rate. Species $v$ is assumed to be an unbiased random walker with constant jump probabilities that are neither affected by its own density nor by the density of the other species. We have 


$$
\left\{\begin{array}{l}
p(u, v)=3.6 u(1-u)+\frac{1}{10} \\
q(u, v)=1-u \\
m(u, v)=1 \\
r(u, v)=1
\end{array}\right.
$$

In the simulation of this aggregation model (Figures 3(a), 3(b)), we see species $v$ smoothly spreading out, as expected. The peak of species $u$ stays at 0.5 for the entire simulation. This is because species $u$ has no preference about which cell to aggregate to, as initially it has constant population densities across all sites it inhabits. The self-diffusion term in the $p$ function leads to species $u$ spreading out slowly from this plateau, however this self-diffusion is partially counteracted by its aggregation strategy. In this model, the aggregation is limited for large values of $u$, which is responsible for the limitation of $u$. In a true aggregation model without such constraint, the entire population would eventually accumulate in one or two neighboring sites (data not shown).

In the last example that we show, the dispersal strategies of both species depend simultaneously on the densities of their own and the other species. Both species are seeking cells which are the least populated. Each species' strategy takes into account the total population of a cell as all the jump probabilities are functions of both $u$ and $v$. Species $v$ spreads at a faster rate because of the coefficients in the exponential of the $r(u, v)$ function. We take

$$
\left\{\begin{array}{l}
p(u, v)=e^{-u-v} \\
q(u, v)=1-e^{-u-v} \\
m(u, v)=e^{-u-v} \\
r(u, v)=1-e^{-10 u-10 v}
\end{array}\right.
$$

This model of spreading species is illustrated in Figures 3(c) and 3(d). Since both species spread by considering the total population density in a cell, there is less crossover of the two species on the lattice in this simulation than in the case where they only self-diffuse. Once the two populations meet in the center of the lattice, they each begin to more strongly spread away from the center, as there is already a significant population located there (i.e. the other species).

\section{Deriving macroscopic models by passing to the continuous limit, $h \rightarrow 0$}

In the spatially discrete model (1) the dispersal strategy is described based on the probabilities of individuals to move between neighboring sites. We now will derive a macroscopic model starting from this micro level description. This has been done frequently in the literature for single species models describing a variety of dispersal strategies, e.g. $[1,4,5,6,7,9,10]$. Usually one obtains parabolic partial differential equations in the macroscopic description. In the 


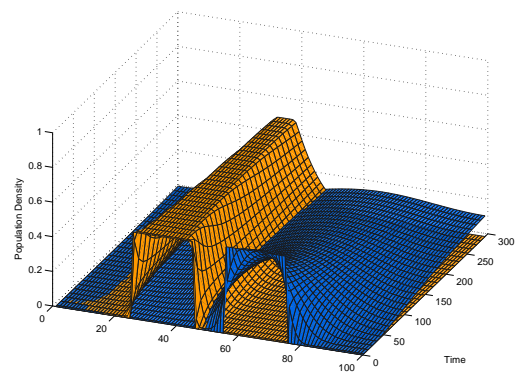

(a) Aggregator and Fickian Diffuser

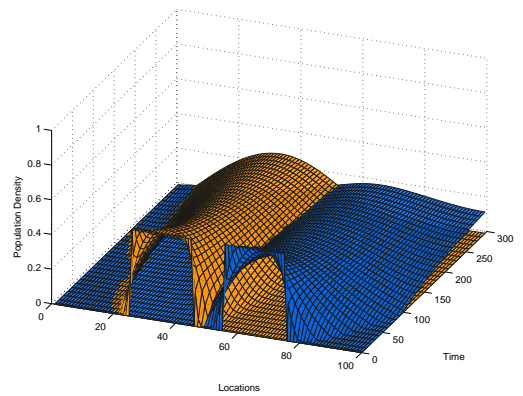

(c) Two Spreading Species

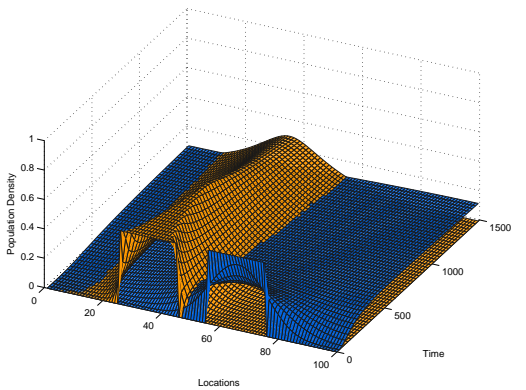

(b) Aggregator and Fickian Diffuser (extended)

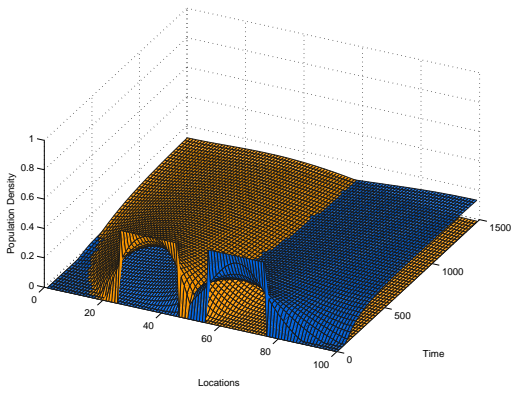

(d) Two Spreading Species (extended)

Figure 3: Simulations using jump probabilities from Section 2. Species $v$ is shaded blue and species $u$ is orange. Figures (a), (b): jump probabilities given by (5), 300 and 1500 time steps, respectively. Figures (c), (d): jump probabilites from (6), 300 and 1500 time steps, respectively.

single species case, if the probability for an individual to move between sites does not depend on the local population density, one obtains a PDE with linear (Fickian) diffusion, otherwise one obtains a nonlinear diffusion equation. The macroscopic partial differential equation models are derived by refining the spatial grid resolution, i.e. one introduces more and more sites, decreasing the distance between neighboring sites, $h \rightarrow 0$.

The starting point is the master equation from (1), 


$$
\begin{aligned}
\frac{\partial u_{i}}{\partial t}= & \mathcal{T}_{i-1}^{+} u_{i-1}+\mathcal{T}_{i+1}^{-} u_{i+1}-\left(\mathcal{T}_{i}^{+}+\mathcal{T}_{i}^{-}\right) u_{i}, \\
& \text { where } \mathcal{T}_{i}^{ \pm}=\alpha q\left(u_{i}, v_{i}\right) p\left(u_{i \pm 1}, v_{i \pm 1}\right), \\
\frac{\partial v_{i}}{\partial t}= & \mathcal{S}_{i-1}^{+} v_{i-1}+\mathcal{S}_{i+1}^{-} v_{i+1}-\left(\mathcal{S}_{i}^{+}+\mathcal{S}_{i}^{-}\right) v_{i}, \\
& \text { where } \mathcal{S}_{i}^{ \pm}=\beta r\left(u_{i}, v_{i}\right) m\left(u_{i \pm 1}, v_{i \pm 1}\right) .
\end{aligned}
$$

We only demonstrate the procedure for (7), as the steps required for (8) are identical. For simplicity of notation, let $q_{i}=q\left(u_{i}, v_{i}\right), p_{i}=p\left(u_{i}, v_{i}\right)$. This allows (7) to be written as

$$
\frac{\partial u_{i}}{\partial t}=\alpha\left(q_{i-1} p_{i} u_{i-1}+q_{i+1} p_{i} u_{i+1}-\left(q_{i} p_{i+1}+q_{i} p_{i-1}\right) u_{i}\right) .
$$

To make the transition from a spatially discrete to a continuous model we first introduce two continuous functions $u(t, x)$ and $v(t, x)$ that interpolate the grid functions $u_{i}(t)$ and $v_{i}(t)$, respectively, i.e.

$$
u\left(t, x_{i}\right)=u_{i}(t), \quad v\left(t, x_{i}\right)=v_{i}(t) .
$$

Assuming sufficient smoothness, we formally expand these two functions around $x_{i}$ in terms of the variable $h$, which is the distance between two neighboring cells. We use $\frac{\partial u_{i}}{\partial x}$ as a short-hand for $\frac{\partial u}{\partial x}\left(t, x_{i}\right)$ and obtain

$$
\begin{aligned}
& u\left(t, x_{i \pm 1}\right)=u_{i}(t) \pm h \frac{\partial u_{i}(t)}{\partial x}+\frac{h^{2}}{2} \frac{\partial^{2} u_{i}(t)}{\partial x^{2}}+\mathcal{O}\left(h^{3}\right) \\
& v\left(t, x_{i \pm 1}\right)=v_{i}(t) \pm h \frac{\partial v_{i}(t)}{\partial x}+\frac{h^{2}}{2} \frac{\partial^{2} v_{i}(t)}{\partial x^{2}}+\mathcal{O}\left(h^{3}\right) .
\end{aligned}
$$

Next, we approximate $q\left(u_{i \pm 1}, v_{i \pm 1}\right)$ and $p\left(u_{i \pm 1}, v_{i \pm 1}\right)$ by $2^{\text {nd }}$ order Taylor polynomials about $u_{i}$ and $v_{i}$,

$$
\begin{aligned}
q\left(u_{i \pm 1}, v_{i \pm 1}\right)= & q\left(u_{i}, v_{i}\right)+\left(u_{i \pm 1}-u_{i}\right) \frac{\partial q}{\partial u}\left(u_{i}, v_{i}\right)+\left(v_{i \pm 1}-v_{i}\right) \frac{\partial q}{\partial v}\left(u_{i}, v_{i}\right) \\
& +\frac{1}{2}\left[\left(u_{i \pm 1}-u_{i}\right)^{2} \frac{\partial^{2} q}{\partial u^{2}}\left(u_{i}, v_{i}\right)+2\left(u_{i \pm 1}-u_{i}\right)\left(v_{i \pm 1}-v_{i}\right) \frac{\partial^{2} q}{\partial u \partial v}\left(u_{i}, v_{i}\right)\right. \\
& \left.+\left(v_{i \pm 1}-v_{i}\right)^{2} \frac{\partial^{2} q}{\partial v^{2}}\left(u_{i}, v_{i}\right)\right] \\
p\left(u_{i \pm 1}, v_{i \pm 1}\right)= & p\left(u_{i}, v_{i}\right)+\left(u_{i \pm 1}-u_{i}\right) \frac{\partial p}{\partial u}\left(u_{i}, v_{i}\right)+\left(v_{i \pm 1}-v_{i}\right) \frac{\partial p}{\partial v}\left(u_{i}, v_{i}\right) \\
& +\frac{1}{2}\left[\left(u_{i \pm 1}-u_{i}\right)^{2} \frac{\partial^{2} p}{\partial u^{2}}\left(u_{i}, v_{i}\right)+2\left(u_{i \pm 1}-u_{i}\right)\left(v_{i \pm 1}-v_{i}\right) \frac{\partial^{2} p}{\partial u \partial v}\left(u_{i}, v_{i}\right)\right. \\
& \left.+\left(v_{i \pm 1}-v_{i}\right)^{2} \frac{\partial^{2} p}{\partial v^{2}}\left(u_{i}, v_{i}\right)\right] .
\end{aligned}
$$


The equations (10), (11), (12), and (13) are then substituted into (7). We obtain

$$
\begin{aligned}
\frac{\partial u_{i}}{\partial t}= & \alpha h^{2} \frac{\partial^{2} u_{i}}{\partial x^{2}}\left[p_{i} \frac{\partial q}{\partial u}\left(u_{i}, v_{i}\right) u_{i}+p_{i} q_{i}-q_{i} \frac{\partial p}{\partial u}\left(u_{i}, v_{i}\right) u_{i}\right] \\
& +\alpha h^{2} \frac{\partial u_{i}^{2}}{\partial x}\left[p_{i} \frac{\partial^{2} q}{\partial u^{2}}\left(u_{i}, v_{i}\right) u_{i}+2 p_{i} \frac{\partial q}{\partial u}\left(u_{i}, v_{i}\right)-q_{i} \frac{\partial^{2} p}{\partial u^{2}}\left(u_{i}, v_{i}\right) u_{i}\right] \\
& +\alpha h^{2} \frac{\partial^{2} v_{i}}{\partial x^{2}}\left[p_{i} \frac{\partial q}{\partial v}\left(u_{i}, v_{i}\right) u_{i}-q_{i} \frac{\partial p}{\partial v}\left(u_{i}, v_{i}\right) u_{i}\right] \\
& +\alpha h^{2} \frac{\partial v_{i}^{2}}{\partial x}\left[p_{i} \frac{\partial^{2} q}{\partial v^{2}}\left(u_{i}, v_{i}\right) u_{i}-q_{i} \frac{\partial^{2} p}{\partial v^{2}}\left(u_{i}, v_{i}\right) u_{i}\right] \\
& +\alpha h^{2} \frac{\partial u_{i}}{\partial x} \frac{\partial v_{i}}{\partial x}\left[2 p_{i} \frac{\partial^{2} q}{\partial u \partial v}\left(u_{i}, v_{i}\right) u_{i}+2 p_{i} \frac{\partial q}{\partial v}\left(u_{i}, v_{i}\right)-2 q_{i} \frac{\partial^{2} p}{\partial u \partial v}\left(u_{i}, v_{i}\right) u_{i}\right]+\mathcal{O}\left(h^{3}\right) .
\end{aligned}
$$

Dropping $\mathcal{O}\left(h^{3}\right)$ terms and passing to the continuous limit, $h \rightarrow 0$, and rearranging the order of terms we obtain

$$
\left\{\begin{array}{l}
\frac{\partial u}{\partial t}=\alpha_{0} \frac{\partial}{\partial x}\left(p q u_{x}+u\left(p \frac{\partial q}{\partial u}-q \frac{\partial p}{\partial u}\right) u_{x}+u\left(p \frac{\partial q}{\partial v}-q \frac{\partial p}{\partial v}\right) v_{x}\right) \\
\frac{\partial v}{\partial t}=\beta_{0} \frac{\partial}{\partial x}\left(m r v_{x}+v\left(m \frac{\partial r}{\partial v}-r \frac{\partial m}{\partial v}\right) v_{x}+v\left(m \frac{\partial r}{\partial u}-r \frac{\partial m}{\partial u}\right) u_{x}\right) .
\end{array}\right.
$$

The reaction-diffusion equation for $v$ is derived identically.

Note that in (15) the probabilities $q, p$ of individuals of species $u$ to move into neighboring sites depends on the density of the individuals of species $v$. This introduces cross-diffusion terms. Cross-diffusion vanishes locally as $u$ vanishes, which is a necessary condition for cross-diffusion systems to guarantee positivity of solutions [3]. Due to the specific structure of the cross-diffusion terms, these can also be understood as convective transport terms for species $u$ where the transport velocity $-\alpha_{0}^{-1}\left(p \frac{\partial q}{\partial v}-q \frac{\partial p}{\partial v}\right) v_{x}$ depends on the gradient of other species. If $p$ and $q$ do not depend explicitly on $u$, e.g. in the case scenarios in the previous section, this convective velocity only depends on the other species. If $p$ and $q$ do

not depend on the other species, as in the aggregator example in the previous section, the cross-diffusion effect is eliminated.

\section{Inverse problem}

We investigate now whether certain macroscopic PDE models for dispersal of two interacting species that can be found in the literature can be derived from our microscopic master equation (1), which is based on a dependency of $p$ and $q$ on the densities of both species in the arrival and departure site. We accomplish this by finding appropriate functions $m, p, q, r$.

The first example for which we carry out this analysis is the case where both species disperse according to linear, or Fickian, diffusion. This is undoubtedly the most frequently used model to describe two interacting species. In this model the dispersion of the species does not depend on the densities of either 
species. In Fickian diffusion, the dispersal flux of a species is proportional to the negative of the density gradient, i.e. gradients are smoothly obliterated.

Another model that has been frequently used in the literature is the crossdiffusion model of Shigesada-Kawasaki-Teramoto [8]. For the single species case this model has been originally derived from a microscopic behavior description which differs from our approach. For the dual species macroscopic version of this model a derivation from the microscopic behavior has not been given.

The third model that we include is the Chattopadhyay-Chatterjee model [2] which was originally formulated for two competing species. Also for this model, a derivation of the macroscopic model from a microscopic description has not been given.

\subsection{Fickian Diffusion}

The first of these models we attempt to recover is Fickian diffusion. Fickian diffusion is the random diffusion of a species which spreads out from higher concentrations to lower concentrations. The model is written

$$
\left\{\begin{array}{l}
\frac{d u}{d t}=D_{1} \frac{\partial^{2} u}{\partial x^{2}}, \\
\frac{d v}{d t}=D_{2} \frac{\partial^{2} v}{\partial x^{2}},
\end{array}\right.
$$

where the constants $D_{1}$ and $D_{2}$ are positive self-diffusion coefficients for species $u$ and $v$ respectively.

For (16) to be recovered from (15) by the jump probabilities $p, q, m$, and $r$, the equations that must be satisfied are

$$
\begin{aligned}
D_{1} / \alpha_{0} & =p q+u\left(p q_{u}-q p_{u}\right) \\
p q_{v} & =q p_{v} \\
D_{2} / \beta_{0} & =m r+v\left(m r_{v}-r m_{v}\right) \\
m r_{u} & =r m_{u}
\end{aligned}
$$

Clearly, the simplest jump probabilities that can be used to recover Fickian diffusion are constant functions. Then, since all partial derivatives of the jump probabilities are zero, the only equalities to be satisfied are $D_{1}=\alpha_{0} p q$, and $D_{2}=\beta_{0} m r$. This can be accomplished by the constant functions

$$
\left\{\begin{array}{l}
q=p=\sqrt{D_{1} / \alpha_{0}}, \\
m=r=\sqrt{D_{2} / \beta_{0}},
\end{array}\right.
$$

for example.

Since these jump probabilities do not vary with respect to the concentrations of $u$ or $v$ in either the arrival or departure site, neither species has any preference as to where it moves along the lattice. However, due to the nature of the master 


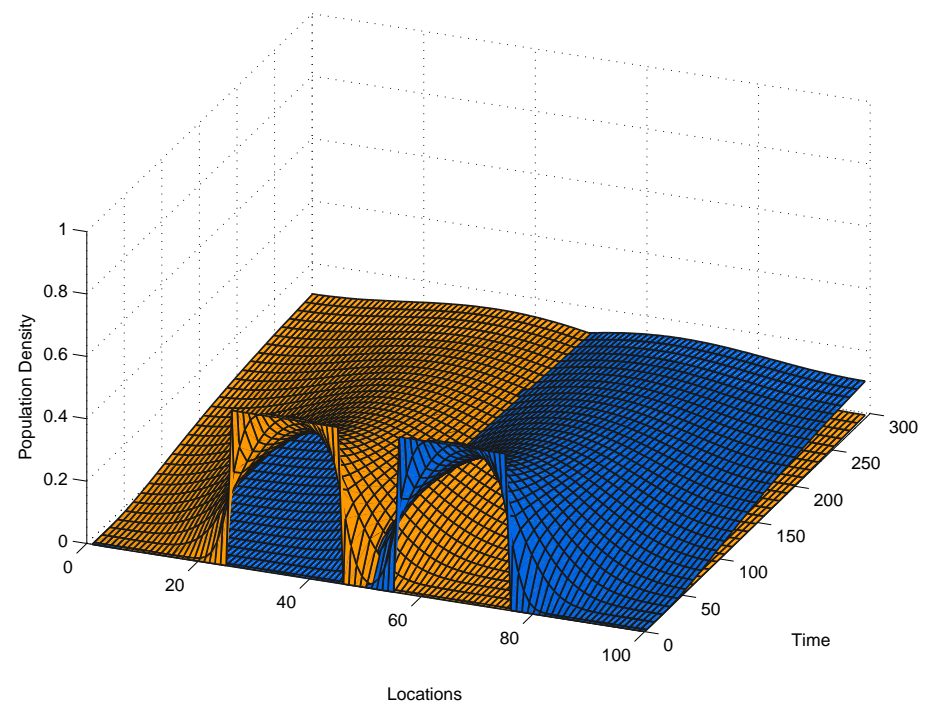

Figure 4: An illustration demonstrating the effect of Fickian diffusion. A diffusion coefficient of 1 is used for each species which relates to jump probabilities $p=q=m=r=1$, from (21). As expected, the simulation shows both species diffusing from places of high density to places of low density. This occurs symmetrically between both species, and neither species is affected by the movements of the other. The initial conditions and 100-cell grid are also the same as were used in Section 2 as given in (2). Species $v$ is shaded blue and species $u$ is orange. 
equation, the higher the volume in a particular cell, the higher the amount of a species will leave the cell. This leads to each species spreading itself out along the lattice, moving from cells with higher densities to cells with lower densities.

The constant functions $p$ and $q$ are the most obvious and simplest jump probabilities to recover Fickian diffusion, but not the only ones. In the general case, an expression for $q(u, v)$ is obtained by solving (18) as an ordinary differential equation in the independent variable $v$ where $u$ is considered to be a constant parameter. This yields $q(u, v)=e^{f(u)} p(u, v)$ where $f(u)$ is formally an arbitrary function of $u$. If $f$ is chosen such that $f^{\prime}(u) \neq \frac{-1}{u}$ for all $u$ in $(0,1]$, then substituting this expression into (17) gives functions for $p$ and $q$

$$
\begin{aligned}
& p(u, v)=\sqrt{\frac{D_{1} / \alpha_{0}}{e^{f(u)}\left(1+u f^{\prime}(u)\right)}}, \\
& q(u, v)=\sqrt{\frac{e^{f(u)} D_{1} / \alpha_{0}}{1+u f^{\prime}(u)}} .
\end{aligned}
$$

The functions $m(u, v)$ and $r(u, v)$ are solved similarly, and found to be

$$
\begin{aligned}
m(u, v) & =\sqrt{\frac{D_{2} / \beta_{0}}{e^{g(v)}\left(1+v g^{\prime}(v)\right)}}, \\
r(u, v) & =\sqrt{\frac{e^{g(v)} D_{2} / \beta_{0}}{1+v g^{\prime}(v)}},
\end{aligned}
$$

where $g(v)$ is an arbitrary function of $v$ and $g^{\prime}(v) \neq \frac{-1}{v}$. The choice of constant functions for $f(u)$ and $g(v)$ leads to all jump probabilities being constant functions.

However, it is easy to verify that these non-constant choices of $m, p, q, r$ lead to a linear diffusion system. Note that $p$ and $q$ do not depend on $v$. Therefore, no cross-diffusion effects are introduced.

\subsection{The cross diffusion model of Shigesada, Kawasaki, and Teramoto}

The next model we investigated is from Shigesada et al [8]. In this paper, the effect of diffusion on pattern formation between two competing species is again considered. The model in its original form also contains an environmental potential function of the heterogenous environment considered in the paper. For the purposes of this paper, the environmental potential function will be ignored (we assume a homogeneous environment) and jump probabilities will be found to recover the diffusion terms alone. The partial differential equation used in the paper without the environmental potential function is 


$$
\left\{\begin{array}{l}
\frac{d u}{d t}=\frac{\partial}{\partial x}\left(D_{11} u_{x}+2 D_{12} u u_{x}+D_{13} u_{x} v+D_{13} u v_{x}\right) \\
\frac{d v}{d t}=\frac{\partial}{\partial x}\left(D_{21} v_{x}+2 D_{23} v v_{x}+D_{22} u_{x} v+D_{22} u v_{x}\right)
\end{array}\right.
$$

so to recover (22) from (15), the jump probabilities must satisfy

$$
\begin{aligned}
D_{11}+2 D_{12} u+D_{13} v & =p q+u\left(p q_{u}-q p_{u}\right), \\
D_{13} & =\left(p q_{v}-q p_{v}\right), \\
D_{21}+2 D_{23} v+D_{22} u & =m r+v\left(m r_{v}-r m_{v}\right), \\
D_{22} & =\left(m r_{u}-r m_{u}\right) .
\end{aligned}
$$

Here we implicitly included $\alpha_{0}$ and $\beta_{0}$ in the coefficients $D_{i j}$.

Proposition 1. The Shigesada-Kawasaki-Teramoto model can be recovered from the master equation (1) with polynomial jump probability functions.

Proof. We make the quadratic ansatz

$$
\begin{aligned}
& p(u, v)=a_{1}+a_{2} u+a_{3} u^{2}+a_{4} v+a_{5} v^{2}+a_{6} u v \\
& q(u, v)=b_{1}+b_{2} u+b_{3} u^{2}+b_{4} v+b_{5} v^{2}+b_{6} u v
\end{aligned}
$$

where the coefficients $a_{i}, b_{i}$ are constants. We substitute this into (15) and compare coefficients. Then, the equation that must be satisfied, from (23) and $(24)$, is

$$
\begin{aligned}
D_{11}+2 D_{12} u+\left(p q_{v}-q p_{v}\right) v & =p q+u\left(p q_{u}-q p_{u}\right) \\
D_{11}+2 D_{12} u & =p q+u\left(p q_{u}-q p_{u}\right)-\left(p q_{v}-q p_{v}\right) v \\
D_{11}+2 D_{12} u & =q\left(p-u p_{u}+v p_{v}\right)+p\left(u q_{u}-v q_{v}\right) .
\end{aligned}
$$

Substituting the quadratic functions for $p$ and $q$, the constants $a_{i}$ and $b_{i}$ are obtained by comparison to yield the solution

$$
\begin{aligned}
& p(u, v)=a_{1}+\frac{a_{3} D_{11}}{D_{12}} u+a_{3} u^{2}+\frac{a_{3} D_{13}}{D_{12}} u v, \\
& q(u, v)=\frac{D_{11}}{a_{1}}+\frac{D_{12}}{a_{1}} u+\frac{D_{13}}{a_{1}} v
\end{aligned}
$$

where $a_{1}$ and $a_{3}$ are arbitrary positive constants. Similar calculations for the functions $m(u, v)$ and $r(u, v)$ show that 


$$
\begin{aligned}
& m(u, v)=a_{1}^{\prime}+\frac{a_{3}^{\prime} D_{21}}{D_{23}} v+a_{3}^{\prime} v^{2}+\frac{a_{3}^{\prime} D_{22}}{D_{23}} u v, \\
& r(u, v)=\frac{D_{21}}{a_{1}^{\prime}}+\frac{D_{22}}{a_{1}^{\prime}} u+\frac{D_{23}}{a_{1}^{\prime}} v,
\end{aligned}
$$

where $a_{1}^{\prime}$ and $a_{3}^{\prime}$ are arbitrary constants. This requires $D_{12}$ and $D_{23}$ to be non-zero.

Another solution, without this constraint, can be obtained by making a linear ansatz for $m, p, q, r$. Again, by comparing coefficients, we find that the only solution is

$$
\left\{\begin{array}{l}
p(u, v)=a_{1}, \\
q(u, v)=\frac{1}{a_{1}}\left(D_{11}+D_{12} u+D_{13} v\right), \\
m(u, v)=a_{1}^{\prime}, \\
r(u, v)=\frac{1}{a_{1}^{\prime}}\left(D_{21}+D_{22} u+D_{23} v\right),
\end{array}\right.
$$

where $a_{1}$ and $a_{1}^{\prime}$ are arbitrary constants.

This proof shows that the Shigesada-Kawasaki-Teramoto model can be interpreted in two ways: as a model in which dispersal depends on both the population densities in the arrival and departure sites, but also as a model in which dispersal only depends on the population densities in the arrival site but not on the departure site. In the next section we shall study a model where this is not possible.

In order to obtain cross-diffusion effects at least one of $p$ and $q$ must depend on $v$. The derivatives of the function $q$ with respect to both $u$ and $v$ are positive, indicating that species $u$ preferentially seeks cells with lower concentrations of both species $u$ and $v$. The values of $D_{12}$ and $D_{13}$ determine the relative importance of species $u$ seeking cells with low concentrations of species $u$ or $v$, respectively. The derivatives of $r$ with respect to both $u$ and $v$ are also positive, meaning species $v$ has the same strategy, with the relative importance of lower concentrations of species $u$ and $v$ given by $D_{22}$ and $D_{23}$, respectively.

A simulation can be run using either of the jump probabilities obtained in Proposition 1. Since both sets of jump probabilities lead to the same diffusion coefficients, the behavior of species $u$ and $v$ will be identical assuming the simulation is run with a sufficiently large number of cells on the lattice. For this reason a simulation is only run using the arrival site independent jump probabilities given by (27) (Figure 5). For the simulation, we let $a_{1}=a_{1}^{\prime}=1$ and choose the $D_{i j}$ 's such that the jump probabilities are

$$
\left\{\begin{array}{l}
p(u, v)=1 \\
q(u, v)=0.1+0.75 u+0.15 v \\
m(u, v)=1 \\
r(u, v)=0.1+0.75 u+0.15 v
\end{array}\right.
$$


These values keep all the jump probabilities bounded between 0 and 1, and create an interesting interaction between the two species. Both species have a small element of random diffusion, and each species preferentially seeks cells with lower concentrations. However, each species weighs the concentration of species $u$ more strongly in their decision making. In the simulation, since each species spreads out heavily weighing the densities of species $u$, we see $u$ spreading in a nearly Fickian manner. Species $v$ spreads more slowly (and hence has a higher peak) because the density of species $u$ is relatively small in all the cells it inhabits, causing the $r$ function to return small values and slow down diffusion.

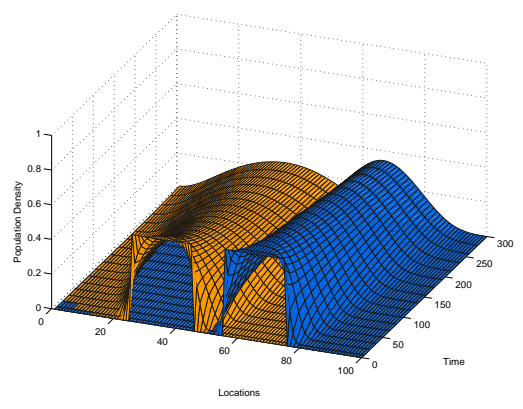

(a) Shigesada Simulation

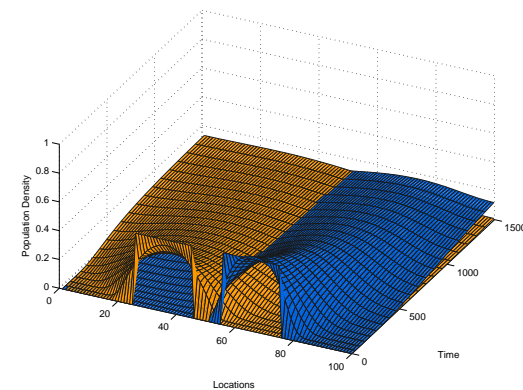

(b) Shigesada Simulation (extended)

Figure 5: 300 and 1500 time step simulations using the departure site independant jump probabilities from the Shigesada et al cross diffusion model (28). The initial conditions and 100-cell grid are also the same as were used in Section 2 as given in (2). Species $v$ is shaded blue and species $u$ is orange.

\subsection{The cross diffusion model of Chattopadhyay and Chat- terjee}

The last model we investigate is from Chattopadhyay and Chatterjee [2]. In this paper, species $v$ spreads by Fickian diffusion with diffusion coefficient $D_{22}$, while the other species, $u$, is modeled by a cross-diffusion term, $D_{12}(u)$, which vanishes if $u$ vanishes. Species $u$ also spreads by Fickian diffusion with diffusion coefficient $D_{11}$. The original model reads

$$
\begin{aligned}
& \frac{d u}{d t}=D_{11} \frac{\partial^{2} u}{\partial x^{2}}+\frac{\partial}{\partial x}\left(D_{12}(u) \frac{\partial v}{\partial x}\right)+u\left(c_{1}-a_{11} u-a_{12} v\right), \\
& \frac{d v}{d t}=D_{22} \frac{\partial^{2} v}{\partial x^{2}}+v\left(c_{2}-a_{21} u-a_{22} v\right),
\end{aligned}
$$

where $D_{12}(u)=D_{12}^{\prime} \frac{u}{\epsilon+u}$. So the model can be rewritten without the competitive growth term as 


$$
\left\{\begin{aligned}
\frac{d u}{d t} & =\frac{\partial}{\partial x}\left(D_{11} u_{x}+D_{12}^{\prime} \frac{u}{\epsilon+u} v_{x}\right) \\
\frac{d v}{d t} & =\frac{\partial}{\partial x}\left(D_{22} v_{x}\right)
\end{aligned}\right.
$$

To recover (29) from (15), the equalities which must be satisfied are

$$
\begin{aligned}
D_{11} & =p q+u\left(p q_{u}-q p_{u}\right), \\
\frac{D_{12}^{\prime}}{\epsilon+u} & =\left(p q_{v}-q p_{v}\right), \\
D_{22} & =m r+v\left(m r_{v}-r m_{v}\right), \\
m r_{u} & =r m_{u},
\end{aligned}
$$

where we again include $\alpha_{0}$ and $\beta_{0}$ in the diffusion coefficients $D_{i j}$. Note that the functions $m(u, v)$ and $r(u, v)$ have the same properties as in the case of Fickian diffusion. So one possible solution is $m=r=\sqrt{D_{22}}$ assuming $D_{22} \leq 1$.

The functions $p(u, v)$ and $q(u, v)$ are solved next. In the previous two cases, the simplest solutions were obtained as functions in which the population densities in the arrival site had no effect, $p=$ const. This is not possible here. In fact, we will show that also the reversed assumption, that dispersal only depends on the arrival site but not on the departure site, does not allow us to recover the Chattopadhyay-Chatterjee model.

Proposition 2. (29) cannot be recovered from (15) by taking either $p(u, v)=$ constant or $q(u, v)=$ constant.

Proof. Let $p(u, v)=k$ where $k$ is an arbitrary constant. Then, the two equalities that must be satisfied, from (30) and (31), are

$$
\begin{aligned}
D_{11} & =k q+k u q_{u}, \\
\frac{D_{12}^{\prime}}{\epsilon+u} & =k q_{v} .
\end{aligned}
$$

Equation (34) can be solved as a differential equation for $q$, yielding $q(u, v)=$ $\frac{c_{1}}{u}+\frac{D_{11}}{k}+\frac{1}{u} f(v)$ where $f(v)$ is an arbitrary function of $v$. This expression for $q$ can be substituted into (35), giving the equality $\frac{D_{12}^{\prime} u}{k(\epsilon+u)}=f^{\prime}(v)$ which is a contradiction as $f$ is a function of $v$ only.

Similarly, setting $q(u, v)=n$ where $\mathrm{n}$ is an arbitrary constant gives the equalities

$$
\begin{aligned}
D_{11} & =n p-u n p_{u} \\
\frac{D_{12}^{\prime}}{\epsilon+u} & =-n p_{v}
\end{aligned}
$$

from (30) and (31). 
Equation (36) is solved as a differential equation, giving $p(u, v)=c_{2} u+$ $\frac{D_{11}}{n}-\frac{D_{11}}{n} u g(v)$ where $g(v)$ is an arbitrary function of $v$. This expression for $q$ can be substituted into (35), giving the equality $\frac{D_{12}^{\prime}}{D_{11} u(\epsilon+u)}=g^{\prime}(v)$ which is a contradiction as $g$ is a function of $v$ only.

One possible way to find jump probabilities to recover (29) from (15) is to assume they can be separated into independent functions of $u$ and $v$. We let $p(u, v)=f(u) g(v)$ and $q(u, v)=h(u) j(v)$. Then, the two equalities which need to be satisfied are

$$
\begin{aligned}
D_{11} & =g j\left(f h+u\left(f h^{\prime}-h f^{\prime}\right)\right), \\
\frac{D_{12}^{\prime}}{\epsilon+u} & =f h\left(g j^{\prime}-j g^{\prime}\right),
\end{aligned}
$$

from (30) and (31), respectively. This implies that the following must hold for arbitrary constants $\lambda_{1}$ and $\lambda_{2}$ :

$$
\begin{aligned}
g j^{\prime}-j g^{\prime} & =\lambda_{1}, \\
g j & =\lambda_{2}, \\
f h & =\frac{D_{12}^{\prime}}{\lambda_{1}(\epsilon+u)}, \\
f h+u\left(f h^{\prime}-h f^{\prime}\right) & =\frac{D_{11}}{\lambda_{2}} .
\end{aligned}
$$

The functions $g(v)$ and $j(v)$ are solved first. Expressions for $g$ and $g^{\prime}$ are obtained from (39) and substituted into (38), giving the ordinary differential equation $j^{\prime}=\frac{\lambda_{1}}{2 \lambda_{2}} j$. The functions can then be solved and are given as

$$
\begin{aligned}
& j(v)=c_{1} \exp \left(\frac{\lambda_{1}}{2 \lambda_{2}} v\right), \\
& g(v)=\frac{\lambda_{2}}{c_{1}} \exp \left(\frac{-\lambda_{1}}{2 \lambda_{2}} v\right),
\end{aligned}
$$

where $c_{1}$ is an arbitrary constant.

Next, the functions $f(u)$ and $h(u)$ are solved. Expressions for $f$ and $f^{\prime}$ are obtained from (40) and substituted into (41), giving the ordinary differential equation $h^{\prime}=h\left(\frac{D_{11} \lambda_{1}(\epsilon+u)}{2 \lambda_{2} D_{12}^{\prime} u}-\frac{1}{2 u}-\frac{1}{2(\epsilon+u)}\right)$. From this, $h(u)$ is solved, after which $f(u)$ is solved from (40). The expressions for $f(u)$ and $h(u)$,

$$
\begin{aligned}
& h(u)=c_{2} e^{c_{3}}(\epsilon+u)^{-\frac{1}{2}} u^{\frac{D_{11} \lambda_{1} \epsilon-\lambda_{2} D_{12}^{\prime}}{2 \lambda_{2} D_{12}^{\prime}}} \exp \left(\frac{D_{11} \lambda_{1}}{2 \lambda_{2} D_{12}^{\prime}} u\right), \\
& f(u)=\frac{D_{12}^{\prime}}{\lambda_{1} c_{2} e^{c 3}}(\epsilon+u)^{-\frac{1}{2}} u^{\frac{\lambda_{2} D_{12}^{\prime}-D_{11} \lambda_{1} \epsilon}{2 \lambda_{2} D_{12}^{\prime}}} \exp \left(-\frac{D_{11} \lambda_{1}}{2 \lambda_{2} D_{12}^{\prime}} u\right),
\end{aligned}
$$


lead to the jump probabilities

$$
\left\{\begin{array}{l}
p(u, v)=\frac{1}{c} \frac{D_{12}^{\prime} \lambda_{2}}{\lambda_{1}}(\epsilon+u)^{-\frac{1}{2}} u^{\frac{1}{2}-\alpha \beta \epsilon} \exp (-\alpha \beta u-\beta v) \\
q(u, v)=c(\epsilon+u)^{-\frac{1}{2}} u^{\alpha \beta \epsilon-\frac{1}{2}} \exp (\alpha \beta u+\beta v), \\
m(u, v)=\sqrt{D_{22}}, \\
r(u, v)=\sqrt{D_{22}},
\end{array}\right.
$$

where $c=c_{1} c_{2} e^{c_{3}}$ and the substitutions $\alpha=\frac{D_{11}}{D_{12}^{\prime}}$, and $\beta=\frac{\lambda_{1}}{2 \lambda_{2}}$, have been made for clarity.

The qualitative properties of these functions depend heavily on the values chosen for the parameters, and the $q$ function blows up for some choices of parameters as $u \rightarrow 0$. Fortunately, the blow-up in $q$ is dampened by a simultaneous vanishing of $p$, and the overall jump probability $p q$ is bounded. One obtains for the dispersal rate in the continuous limit $\mathcal{T}_{i}^{ \pm}=D_{12}^{\prime} \frac{\lambda_{2}}{\lambda_{1}}(\epsilon+u)^{-1}$.

A simulation is run using these jump probabilities (Figure 6). The jump probabilities obtained in the inverse problem of this model present some problems since the $q$ function can blow up as $u \rightarrow 0$. For this reason, the population densities of species $u$ are kept well above zero, and values of the variables are chosen carefully. The exact initial conditions are taken to be

$$
\left\{\begin{array}{l}
u_{i}(0)=0.75, \forall i \\
v_{i}(0)=0.5,0 \leq i \leq 25,46 \leq i \leq 55,76 \leq i \leq 100 \\
v_{i}(0)=0.5,26 \leq i \leq 45,56 \leq i \leq 75 .
\end{array}\right.
$$

Values for the parameters are chosen so that all the jump probabilities are between zero and one as long as the density of species $u$ is above 0.5 in all sites on the lattice:

$$
\begin{aligned}
D_{11} & =0.4, \\
D_{12}^{\prime} & =0.1, \\
D_{22} & =0.01, \\
c & =0.03, \\
\epsilon & =0.001, \\
\lambda_{1} & =\lambda_{2}=1 .
\end{aligned}
$$

These values give the jump probabilities

$$
\left\{\begin{array}{l}
p(u, v)=\frac{10}{3}(0.001+u)^{-\frac{1}{2}} u^{0.48} e^{-2 u-0.5 v} \\
q(u, v)=\frac{3}{100}(0.001+u)^{-\frac{1}{2}} u^{-0.48} e^{2 u+0.5 v} \\
m(u, v)=0.1 \\
r(u, v)=0.1
\end{array}\right.
$$

from (42). 


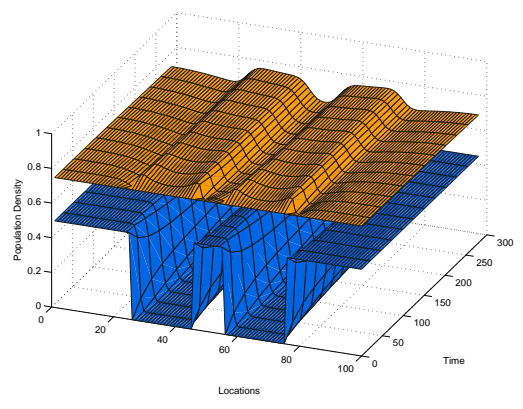

(a) Chattopadhyay and Chatterjee

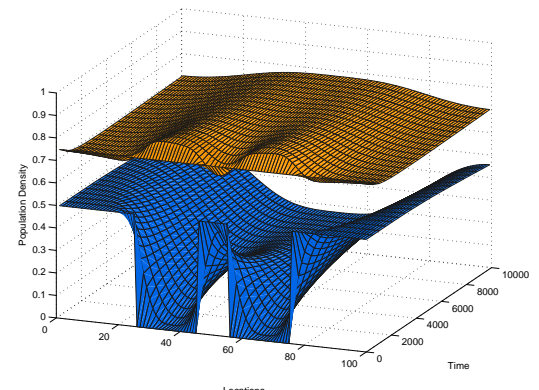

(b) Chattopadhyay and Chatterjee (extended)

Figure 6: Simulations using jump probabilities from the Chattopadhyay and Chatterjee model (44) for 300 and 10000 time steps. The initial conditions are given by (43). Species $v$ is shaded blue and species $u$ is orange.

The simulation shows species $v$ spreading slowly by Fickian diffusion, and species $u$ aggregating in places with low concentrations of species $v$, as expected. The self diffusion of species $u$ is less apparent in this simulation due to the nature of the initial conditions.

\section{Conclusions}

We used the spatially discrete master equation to derive a macro scale model governed by a set of two partial differential equations, which were found to contain cross diffusion terms. We were able to show that Fickian diffusion, as well as the models in [2] and [8] were special cases of this macro scale model. That is, these macro scale models commonly used to model dual species interaction can be derived from the local rules of a micro scale spatially discrete master equation model.

In the future, the approach in the inverse problem could likely be used to find jump probabilities which recover many other reaction-diffusion systems. The model used in this paper could also be extended further by increasing the number of spatial dimensions from one to two. In addition, growth terms could also be included, such as those used in predator-prey, competition, and chemotaxis models.

\section{References}

[1] K. ANGUIGE, C. SCHMEISER, A one-dimensional model of cell diffusion and aggregation, incorporating volume filling and cell-to-cell adhesion, J. Math. Biol., 58(2009), pp. 395-427. 
[2] J. CHATTOPADHYAY, S. CHATTERJEE, Cross diffusional effect in a Lotka-Volterra competitive system, Nonlinear Phenom. Complex Syst., 4(2001), pp. 364-369.

[3] M. A. EFENDIEV, S. SONNER, On verifying mathematical models with diffusion, transport and interaction, Advances in Math. Sci. Appls. 32(2010), pp. $41-67$

[4] H. KHASSEHKHAN, T. HILLEN, H. J. EBERL, A Nonlinear Master Equation for a Degenerate Diffusion Model of Biofilm Growth, Lecture Notes in Comput. Sci., 5544(2009), pp. 735-744.

[5] M. LIZANA, V. PADORN, A spatially discrete model of aggregating populations, J. Math. Biol., 38(1999), pp. 79-102.

[6] A. OKUBO, S. A. LEVIN, Diffusion and Ecological Problems: Modern Perspectives, 2nd ed., Springer, New York, NY, 1980.

[7] K. PAINTER, T. HILLEN, Volume-Filling and Quorum Sensing in Models for Chemosenstive Movement, Can. Appl. Math. Q., 10(2002), pp. 501-543.

[8] N. SHIGESADA, K. KAWASAKI, E. TERAMOTO, Spatial Segregation of Interacting Species, J. Theoret. Biol., 79(1978), pp. 83-99.

[9] S. TURnER, J. A. SHERRATT, K. J. PAINTER, From a discrete to a continuous model of biological cell movement, Phys. Rev E, 69(2004), pp. $1-10$.

[10] A. W. VISSER, Lagrangian modelling of plankton motion: From deceptively simple random walks to Fokker-Planck and back again, J. Mar. Sys., 70(2008), pp. 287-299. 